Bundesgesundheitsbl -

Gesundheitsforsch - Gesundheitsschutz

2000 • 43:343-345 @ Springer-Verlag 2000
Leitthema: Umwelt und Gesundheit

U. Kaiser

Robert Koch-Institut, Berlin

\section{Zusammenfassung}

Das Aktionsprogramm „Umwelt und Gesundheit" (APUG) hat es bisher noch nicht geschafft, in die Wahrnehmung der Öffentlichkeit und der Medien zu gelangen. Auf der Fachebene wurden bisher nur die komplexen Zusammenhänge und übergeordneten Perspektiven thematisiert. Neben weiteren Maßnahmen soll ein moderierter elektronischer Diskurs zu den Umsetzungsmöglichkeiten des APUG eine breite inhaltliche Beteiligung der Öffentlichkeit fördern, konkrete Aktivitäten auf der lokalen Ebene initiieren und somit insgesamt den notwendigen Praxisbezug herstellen.

\section{Schlüsselwörter}

Aktionsprogramm Umwelt und Gesundheit . Beteiligung der Öffentlichkeit . Nicht-Regierungsorganisationen (NRO)

\title{
Elektronischer Diskurs zu Umsetzungsmöglichkeiten des Aktionsprogramms "Umwelt und Gesundheit"
}

$D_{\text {i }}$ praktische Umsetzung der „National Environmental Health Action Plans" (NEHAPs) kann aus der Sicht der WHO nur auf der Basis der PIPP-Strategie (public information and public participation) erfolgreich durchgeführt werden. In einem mehrjährigen Prozess wurde unter der Federführung des Umwelt- und des Gesundheitsministeriums das deutsche „Aktionsprogramm Umwelt und Gesundheit" (APUG) konzipiert, zu dem eine umfangreiche Dokumentation in Form eines Sachstandsberichtes erstellt wurde. Beide Papiere wurden noch rechtzeitig vor der Europäischen Ministerkonferenz im Juni 1999 publiziert. Sowohl im Aktionsprogramm als auch in der Dokumentation werden umzusetzende Maßnahmen notwendigerweise in sehr komplexen Zusammenhängen und übergeordneten Perspektiven thematisiert. In dem bisherigen Prozess wurde die Einbindung der Nichtregierungsorganisationen (NROs) nicht im nötigen Umfang realisiert. Dies führte u.a. dazu, dass aus den Reihen der NROs ein eigenes Diskussionspapier zum APUG publiziert wurde. Um in Zukunft eine weitere parallele Entwicklung zu vermeiden, soll der nun beginnende Prozess der Realisierung/Umsetzung des Aktionsprogramms unter verbindlicher Beteiligung der NROs erfolgen. Seitens der NROs entstand ein Bündnis Umwelt und Gesundheit zur aktiven Unterstützung des APUG.

\begin{abstract}
„Mittels moderner elektronischer Informationssysteme können und sollen sich Nichtregierungsorganisationen an der Umsetzung des Aktionsprogramms beteiligen."
\end{abstract}

Um das Aktionsprogramms aus seinem bisherigen "Schattendasein" zu befreien, haben BMU und BMG die Erarbeitung und Publikation einer öffentlichkeitswirksamen Gesamtdarstellung veranlasst. Die Auseinandersetzung mit dem APUG soll auf breiter Ebene intensiviert und effektiver gestaltet werden. Hierfür ist u.a. auch die Nutzung moderner elektronischer Informations- und Kommunikationssysteme geplant.

\section{Ein elektronischer Diskurs zum APUG}

In einem elektronischen Diskurs können orts- und zeitunabhängig Ideen zusammengetragen werden, kritische Diskussionen vor bzw. mit einem großen elektronischen Publikum stattfinden, konsensuale Positionen erarbeitet und das „Herunterbrechen des APUG auf die lokale Ebene" vorbereitet werden. Eine gemeinsame elektronische Plattform

Dr. Uwe Kaiser

Robert Koch-Institut, Postfach 650280 13302 Berlin 
Bundesgesundheitsbl -

Gesundheitsforsch - Gesundheitsschutz

$2000 \cdot 43: 343-345$ @ Springer-Verlag 2000

\section{U.Kaiser}

Construction of a reliable electronic information tool with the aim to improve the performance of the program „Environment and Health" in the Federal Republic of Germany

\section{Summary}

Until today the "Environment and Health" action program (APUG = Aktionsprogramm "Umwelt und Gesundheit") is not well known, neither by the public nor by the media. At the expert level only complex relationships and general points of view have been discussed. Among other actions an evaluated electronic information tool with the aim to facilitate the tasks to be performed by APUG should promote a broad public participation and initiate concrete actions on the local level. Thus it will provide the essential flow of information between the public and Environmental Health professionals.

\section{Keywords}

National Environmental Health Action Plan (NEHAP) · public participation non-governmental organisation (NGO)

\section{Leitthema: Umwelt und Gesundheit}

wäre auch eine wirksame Unterstützung der Arbeit des NRO-Bündnisses. Der dort vorhandene große Kommunikationsbedarf und der notwendige komplexe Informationsaustausch erfordern zwingend die Einbindung moderner elektronischer Hilfsmittel.

Am geplanten elektronischen Netzwerk (Intranet) können interessierte Kreise/gesellschaftlich relevante Gruppen teilnehmen und sich inhaltlich an der Umsetzung des Aktionsprogramms beteiligen. Zur technischen Realisierung des Intranets wird empfohlen, auf den Erfahrungen des seit Jahren erfolgreich arbeitenden „Umweltmedizinischen Informationsforums UmInfo" aufzubauen. Dieser Informationsverbund hat sich inzwischen zum Standard in der Umweltmedizin entwickelt. Um eine Netzwerkarchitektur mit aktivem „Eigenleben” für das Aktionsprogramm aufzubauen, soll auf einem entsprechenden Server (FirstClass ${ }^{\circledR}$-Intranet) eine vorbereitete elektronische Umgebung geschaffen werden, die den Nutzern folgende Bereiche zur Verfügung stellt:

D Grundlagentexte (read only; Volltextdokumente; Vereinheitlichung des Basiswissens),

D Querverweise (read only; elektronischer Volltextzugriff auf relevante Hintergrundinformationen),
D Diskussionsecke (Bereich der Nutzeraktivitäten; Statements, Diskussionen etc.).

Durch den Einsatz der kostenlosen Software FirstClass ${ }^{\circledR}$ ist ein elektronischer Diskurs komfortabel und schnell über das Internet erreichbar und kann auch über das Regionalknotennetz ohne Internetprovider zugänglich gemacht werden. Ein weniger komfortabler Zugang ist unter Nutzung üblicher ,Web-Browser” ebenfalls möglich. Die FirstClass ${ }^{\circledR}$ Architektur ermöglicht eine flexible und bedarfsorientierte Gestaltung des Systems (z.B. zugriffsgeschützte Bereiche, Unterstrukturierung etc.). Die Abbildungen zeigen einen Arbeitsentwurf zum elektronischen Diskurs.

Generell soll der Zugriff auf Hintergrundinformationen und elektronische Querverweise öffentlich zugänglich sein. Die Volltextdokumente können zum Lesen per „Mausklick” geöffnet werden, oder aber zur weiteren Verwendung via „download” auf dem eigenen Rechner gesichert (abgespeichert) werden. Zur Vermeidung anonymer oder unseriöser Beiträge soll allerdings die aktive inhaltliche Auseinandersetzung bzw. Beteiligung erst nach einer elektronischen Anmeldung, Identifizierung und Freischaltung der Teilnehmer möglich sein.
Abb.1 Der Weg vom Eingangsbildschirm zu den Grundlagentexten

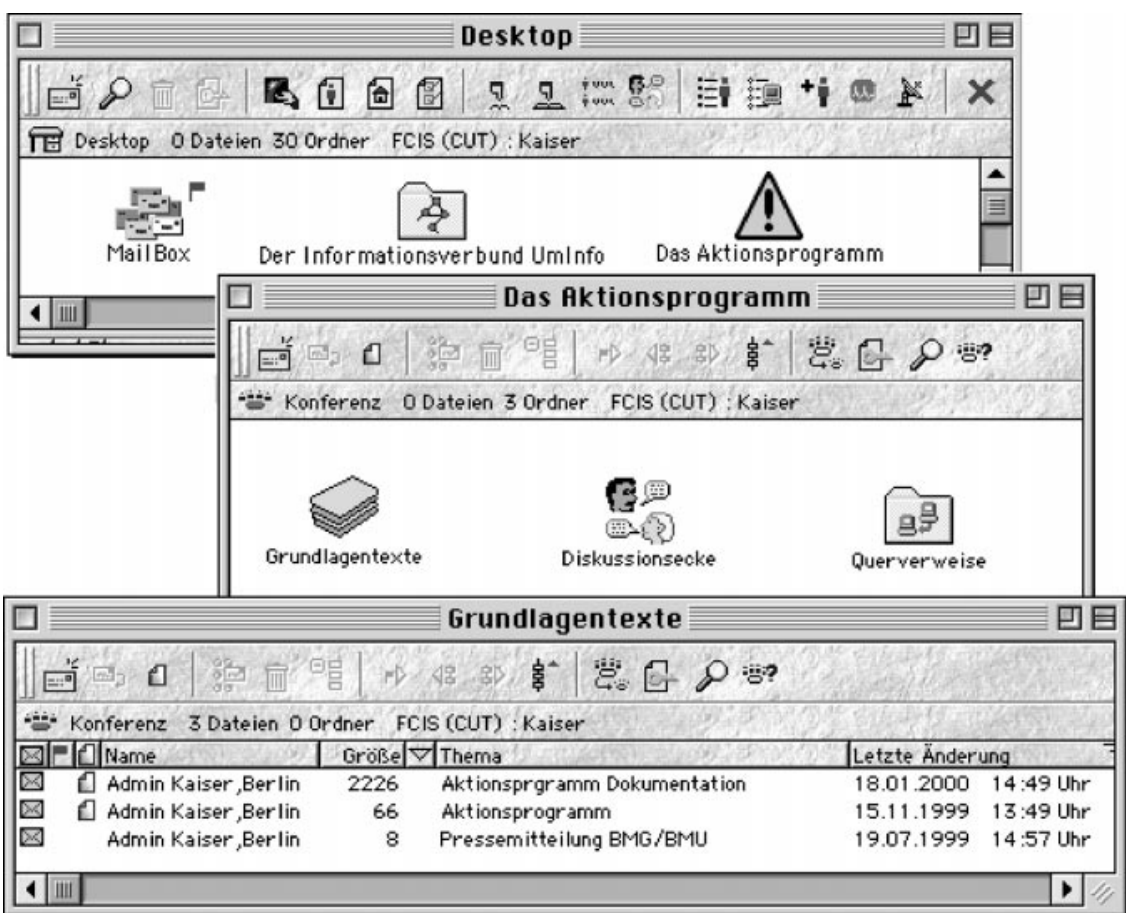




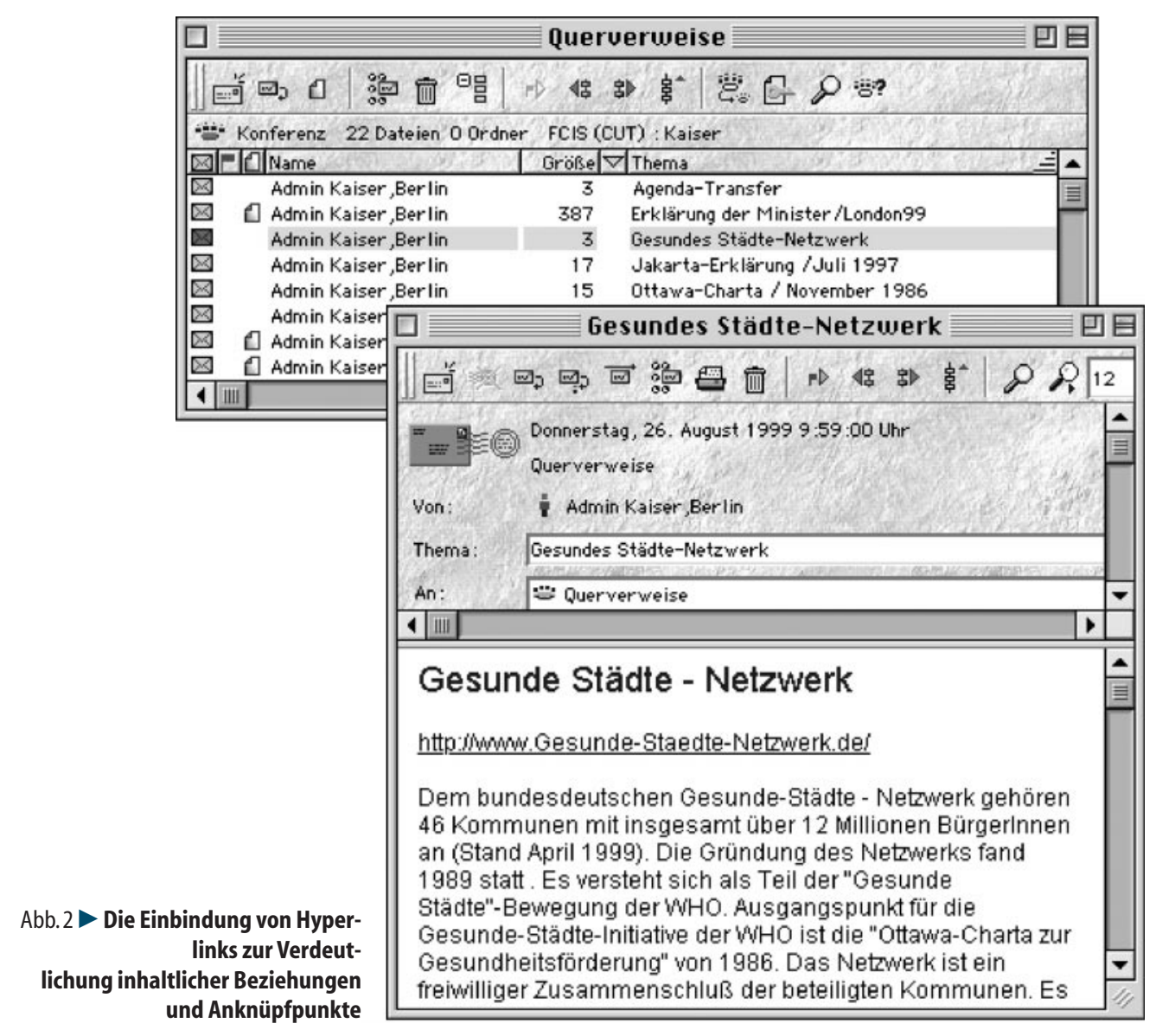

Die Software bietet zahlreiche komfortable Funktionen, die u.a. die aktive Beteiligung im „Diskussionsbereich” erleichtern sollen:

D Verknüpfung zusammengehörender Beiträge zum besseren chronologischen Lesen,

D Antwortfunktion mit Auswahloptionen (nur an Absender, an alle etc.),

D Antworten mit Zitat,

D Erstellen von Mailinglisten,

D Einsatzmöglichkeiten eines Moderators mit privilegierten Rechten,

D elektronische Visitenkarten,

D zum Schutz vor Informationsüberflutung kann für Dokumente ein „Verfallsdatum” festgelegt werden,

Dedarfsorientierte Einrichtung (zugriffsgeschützter) Unterebenen für spezielle Arbeitsgruppen, etc.

Durch die 100\%ige Kompatibilität zum Internet ist das Einbinden elektronischer Querverweise (sog. Hyperlinks) zu anderen relevanten Informationsquellen im Internet problemlos möglich. Aus einem FirstClass ${ }^{\circledR}$-Dokument heraus kann dann der Wechsel zum Internet erfolgen und anschließend wieder der Rücksprung in das Intranet.

Als weitere Service-Leistung soll der Zugriff auf relevante Dokumente im Internet zum Themenkomplex „Umwelt und Gesundheit" erleichtert werden. Hierfür werden in einer Datenbank Kurzbeschreibungen zu den Dokumenten angelegt und mit Hilfe eines strukturierten Vokabulars indexiert. Über menügesteuerte Abfragemasken können dann aus diesem ,"qualitätsgeprüften Informationspool" zur jeweiligen Suchanfrage Trefferlisten mit hoher Relevanz erzeugt werden. Der Überblick über die Kurzbeschreibungen ermöglicht eine weitere Reduzierung der Trefferzahl, um erst danach via Hyperlinkaktivierung das Originaldokument aufzurufen. $\mathrm{Ne}$ ben der Nutzung des Systems als Informationsquelle sollen durch die aktive Beteiligung der Nutzer und durch Aufbau neuer Querverbindungen mit synergistischen Effekten (interdisziplinäre
Kreativität) auch neue Inhalte geschaffen werden. Durch das Vorstellen lokaler Aktivitäten und deren Diskussion hinsichtlich eines „Modellcharakters” könnten Referenzprojekte für einen Katalog konkreter Umsetzungsmaßnahmen zum Aktionsprogramm definiert werden. Der Aufbau internationaler Kontakte zu den verantwortlichen der lokalen Umsetzungsmaßnahmen in anderen Ländern soll einen Erfahrungsaustausch ermöglichen, der ggf. neue Aspekte für diesen Prozess in der Bundesrepublik liefert. Dieser internationale Erfahrungsaustausch sollte unter Einbindung der Kompetenz des WHO-CC am RKI aufgebaut werden, da hier bereits analoge Projekte angesiedelt sind. 